\title{
Test-Retest Reliability, Convergent Validity and Practice Effects of the RBANS in a Memory Clinic Setting: A Pilot Study
}

\author{
Yanhong Dong ${ }^{1,2,3}$, Claire L. Thompson ${ }^{4}$, Shi Huey Joanne Tan², Leon Ben Swie Lim ${ }^{1,2}$, \\ Wanshin Pang ${ }^{1,2}$, Christopher Li-Hsian Chen ${ }^{1,2}$ \\ ${ }^{1}$ Memory Aging and Cognition Centre, Department of Pharmacology, National University Health System, Singapore \\ ${ }^{2}$ Department of Pharmacology, Yong Loo Lin School of Medicine, National University of Singapore, Singapore \\ ${ }^{3}$ Centre for Healthy Brain Ageing and Dementia Collaborative Research Centre, School of Psychiatry, \\ the University of New South Wales, Australia \\ ${ }^{4}$ James Cook University (Australia), Singapore Campus \\ Email: yanhong_dong@nuhs.edu.sg
}

Received June, 2013

\begin{abstract}
This pilot study examined the psychometric properties and clinical utility of a brief neuropsychological instrument (Repeatable Battery for the Assessment of Neuropsychological Status (RBANS). The test-retest reliability, practice effects and convergent validity of RBANS were examined in participants without objective cognitive impairment. The tests were administered at two time points at approximately a two weeks' interval, with 30 cognitively intact participants with a mean age of $63.3 \pm 5.8$ years. Adequate test-retest reliabilities were found for RBANS subtests, index and total scale scores with significant gain scores in immediate memory and visuospatial function. The RBANS showed good convergent validity and the RBANS supplemented with executive and language measures (Colour Trails Test and 30-item modified Boston Naming Test, respectively) demonstrated excellent convergent validity with a formal neuropsychological battery. This pilot study has provided the preliminary evidence of reliability and convergent validity of the RBANS. Additionally, it also provides insight on the practice effects so that clinicians may assess significant changes in RBANS subtests and domain indexes for clinical practice.
\end{abstract}

Keywords: RBANS; Neuropsychological Tests; Test-retest Reliability; Validity; Practice Effect

\section{Introduction}

The prevalence of dementia is estimated to be 35.6 mil-lion patients in 2010 and is expected to double every 20 years [1], thereby creating increasing demand for an efficient, reliable and valid instrument for the early detection of age-related cognitive impairments. This instrument should have the following characteristics: 1) brevity- elderly people with cognitive impairment may only co-operate well with cognitive testing within a short span of time [2]; 2) test-retest reliability- repeated cognitive testing is often required to clarify diagnosis or to monitor progression of symptoms [3]; and 3) demonstrates convergent validity with a formal neuropsychological battery, which covers a range of cognitive domains required for the diagnosis of dementia.

The Repeatable Battery for the Assessment of Neuropsychological Status (RBANS)[4] has demonstrated its reliabilities and validities in the western population and may be considered for the detection of dementia in Sin- gapore. The RBANS is brief and takes approximately 30 minutes [5]. It assesses a total of five cognitive domains with 12 subtests [4]. RBANS has shown sensitivity of $84 \%$ and specificity of $97 \%$ for cognitive impairment in Alzheimer's disease (AD) [6]. However, RBANS was limited in detecting Mild Cognitive Impairment (MCI), as the sum of index scores and six of the subtests (list learning, semantic fluency, coding, list recall, story recall and figure recall) had poor sensitivity (range: $8-24 \%$ ) in detecting MCI[7]. Also, the lack of adequate executive function measures and object naming tasks [8] has been criticized as the drawback of RBANS for differential diagnosis of cognitive impairment in a clinical setting. Therefore, in the current study, we supplemented RBANS with the Color Trails Test (CTT)[9] and the 30-item modified Boston Naming Test (mBNT)[10] to address these drawbacks.

Information on test-retest reliability of the RBANS is limited. Test-retest reliability is first established using cognitively normal participants [11]. A short span of time 
interval (under one month) minimizes the degree to which individuals will go through any physical or psychological changes and therefore is more accurate to assess the reliability of the cognitive instrument [12]. However, short intervals may introduce practice effects. For the RBANS, studies with heterogeneous samples with and without cognitive impairment have reported test-retest reliability of 0.96 over one month in China [13], and of 0.72 over a three month interval in Brazil [14]. Pat-ton et al. [15] reported stable scores over one-year and two-year intervals but test-retest reliability coefficients were not reported. Therefore, this study will evaluate the test-retest reliability of the RBANS and examine its practice effect over a two-week period in Singaporean older adult participants with no objective cognitive impairment (NCI). In addition, the convergent validity of the RBANS will be assessed by the correlation with a locally validated formal neuropsychological battery for Singaporean elderly [16].

The hypotheses of this pilot study were: 1) RBANS and RBANS supplemented with CTT and m-BNT would demonstrate good test-retest reliability over a two-week period; 2) There would be evident practice effects reflected by the gain scores of the individual subtest scores and domain index scores; and 3) RBANS and RBANS supplemented with CTT and m-BNT would demonstrate good convergent validity with a formal neuropsychological battery.

\section{Method}

\subsection{Participants}

We recruited 30 participants with no objective cognitive impairment determined by a formal neuropsychological battery. Of these, 7 were recruited from a memory clinic at the National University Health System (NUHS) in Singapore and a further 23 were volunteers from the community. Inclusion criteria included a minimum age of 50 years and absence of major psychiatric or physical illness and/ or sensory impairment. Two participants delayed their second assessment for 114 days and were therefore excluded from the analyses. This study was approved by National Healthcare Group Domain-Specific Review Board (DSRB), and was conducted in conformity with the Declaration of Helsinki. Written informed consent was obtained from all participants.

\subsection{Measures}

All subtests of the RBANS were scored according to standardized criteria except for Figure Copy and Figure Recall, which were scored according to Duff et al.'s modified criteria [17]. Raw scores of the 12 subtests were converted to age and education adjusted scaled scores [18] before calculating an Index score for each domain. The Sum of Index score was then converted to a Total Scale score, with a mean of 100 and standard deviation of 15 . The Color Trails Test (CTT) $[9,18]$ and 30-item modified Boston Naming Test [10] were administered as supplementary tests to the RBANS[8].

Participants were also assessed using a formal neuropsychological battery locally validated for Singaporean elderly [16]. This battery covers 7 cognitive domains including both non-memory and memory domains. The non-memory domains of the formal neuropsychological battery included: (1) attention (digit span test)[20], visual span test[20] and auditory detection test[21]; (2) language (15-item modified Boston naming[22]) and category fluency[23]; (3) visuomotor speed (symbol digit modalities[24], digit cancellation[25], and mazes[26]; (4) visuoconstruction (visual reproduction subtest of the Wechsler Memory Scale- Revised[20] copy task, clock drawing[27] and the Block Design subtest of the Wechsler Adult Intelligence Scale Revised[28]; (5) executive function (Frontal Assessment Battery[29]). The memory domains of the battery included: (1) verbal memory (word list [10]; and story recall [20]; (2) visual memory (picture recall [20]) and the visual reproduction subtest of the Wechsler Memory Scale-Revised [20].

In addition, routine brief cognitive screening measures, the Abbreviated Mental Test (AMT)[30], the MiniMental State Examination (MMSE)[31] and the Montreal Cognitive Assessment (MoCA)[32] were ad-ministered to all participants. The 15-item Geriatric De-pression Scale (GDS [33]) was administered to screen for depression.

\subsection{Procedure}

The assessments and re-testing were administered by trained research psychologists in accordance with the manual over approximately two weeks.

\subsection{Statistical Analysis}

The descriptive statistics were used to characterize the sample's demographic and clinical profile. Pearson's correlation coefficients were calculated between Time 1 and Time 2 to examine test-retest reliability and convergent validity of the cognitive tests. Practice effects were expressed as effect sizes and calculated by the change in raw subtest scores, index scores and total scale scores between time 1 and time 2 divided by the standard deviation of time 1 scores[34]. This method has been commonly used $[35,36]$.

\section{Results}

\subsection{Sample Characteristics}

Participants had a mean age of $63.3 \pm 5.8$ years with 12.3 
\pm 4.9 years of education (see Table 1 ). Most were Chinese (96.4\%) females (67.9\%). Participants' mean scores of brief cognitive screening tests (AMT, MMSE and MoCA) were within normal range. No participants reported significant symptoms of depression (GDS score: $1.39 \pm 1.62$ ). The mean test-retest interval was approximately two weeks (15.64 \pm 4.72 days).

\subsection{Test-retest Reliability of the RBANS}

The means and standard deviations for the RBANS at each time point, along with the Pearson's correlations are presented in Table 2. Significant correlations were found between times 1 and time 2 raw scores for all subtests except list recall. Further examination of the list recall subtest revealed three outliers. Excluding these outliers led to significant correlations between list recall scores at time 1 and time $2(r=.608, p=.001)$. The additional two tests used to supplement the RBANS (CTT and mBNT) also demonstrated adequate test-retest reliability (see Table 2).

\subsection{Gain Scores and Practice Effects from Time 1 to Time 2}

As shown in Table 2, there were significant gain scores for five of the twelve subtests: list learning and list recall, story memory, figure copy and figure recall with moderate to large effect sizes (.45 - .87). Additionally, significant gain scores were observed in the domain index scores of visuospatial function, immediate memory and delayed memory. There were no significant gain scores in total scale scores of RBANS or the supplementary tests (CTT and mBNT).

Table 1. Demographic characteristics.

\begin{tabular}{lc}
\hline \multicolumn{1}{c}{ Variables } & $N=28$ \\
\hline Age & $63.29(5.84)$ \\
Years education & $12.32(4.87)$ \\
Gender (n, \%) & \\
$\quad$ Males & $9(32.1 \%)$ \\
$\quad$ Females & $19(67.9 \%)$ \\
Ethnicity: & \\
Chinese (n, \%) & $27(96.4 \%)$ \\
Eurasian (n, \%) & $1(3.6 \%)$ \\
AMT & $9.68(0.48)$ \\
MMSE & $27.96(1.45)$ \\
MoCA & $26.64(2.36)$ \\
Retest Interval (days) & $15.64(4.72)$ \\
\hline
\end{tabular}

Values are mean $(S D)$ unless otherwise specified.

Table 2. The test-retest indices of RBANS subtests, supplementary tests (CTT and mBNT), index and total scale scores.

\begin{tabular}{|c|c|c|c|c|c|}
\hline & Time $1 \mathrm{M}$ (SD) & Time 2 M (SD) & Pearson Correlation & Time 2 - Time 1 & Effect Size \\
\hline \multicolumn{6}{|l|}{ Subtests } \\
\hline List learning & $27.18 \quad(5.61)$ & $32.07 \quad(4.97)$ & $0.78^{* * *}$ & $4.89 * * *$ & 0.87 \\
\hline Story memory & $18.82 \quad(3.51)$ & $20.68 \quad(3.57)$ & $0.83^{* * *}$ & $1.86^{* * *}$ & 0.53 \\
\hline Figure copy & $17.46 \quad(2.08)$ & $16.00 \quad(2.67)$ & $0.65 * * *$ & $-1.46^{* * *}$ & -0.70 \\
\hline Line orientation & $16.57 \quad(3.28)$ & $16.29 \quad(3.10)$ & $0.76^{* * *}$ & -0.29 & -0.09 \\
\hline Picture naming & $9.46 \quad(0.79)$ & $9.50 \quad(0.69)$ & $0.71^{* * *}$ & 0.04 & 0.05 \\
\hline Semantic fluency & $18.82 \quad(4.27)$ & $19.14 \quad$ (3.30) & $0.70^{* * *}$ & 0.32 & 0.07 \\
\hline Digit span & $11.29 \quad(2.57)$ & $10.75 \quad$ (2.69) & $0.71^{* * *}$ & -0.54 & -0.21 \\
\hline Coding & $42.11 \quad(9.25)$ & $43.54 \quad(11.22)$ & $0.82^{* * *}$ & 1.43 & 0.15 \\
\hline List recall & $6.25 \quad(3.15)$ & $8.14 \quad(1.58)$ & 0.36 & $1.89 * *$ & 0.60 \\
\hline List recognition & $19.68 \quad(0.55)$ & $19.43 \quad(1.14)$ & $0.47^{*}$ & -0.25 & -0.45 \\
\hline Story recall & $10.18 \quad(1.72)$ & $10.43 \quad(1.60)$ & $0.77^{* * *}$ & 0.25 & 0.15 \\
\hline Figure recall & $12.68 \quad(3.57)$ & $14.29 \quad(3.43)$ & $0.63 * * *$ & $1.61^{* *}$ & 0.45 \\
\hline CTT1 (seconds) & $50.75 \quad(20.00)$ & $47.14 \quad$ (21.11) & $0.57^{* *}$ & -3.61 & -0.18 \\
\hline CTT2 (seconds) & $96.07 \quad$ (30.57) & $89.32 \quad(22.70)$ & $0.67 * * *$ & -6.75 & -0.22 \\
\hline 30-item mBNT & $25.90 \quad(3.64)$ & $26.18 \quad$ (3.69) & $0.90 * * *$ & 0.29 & 0.08 \\
\hline \multicolumn{6}{|l|}{ Indexes } \\
\hline Attention & $94.36 \quad(14.56)$ & $93.25 \quad(14.41)$ & $0.76^{* * *}$ & -1.11 & -0.08 \\
\hline Language & $96.68 \quad(19.80)$ & $98.50 \quad$ (21.19) & $0.55^{* *}$ & 1.82 & 0.09 \\
\hline Visuospatial & $100.96 \quad(13.08)$ & $96.19 \quad(10.16)$ & $0.58^{* *}$ & $-4.77^{*}$ & -0.36 \\
\hline Immediate memory & $100.43 \quad(13.81)$ & $115.90 \quad(11.82)$ & $0.50 *$ & $15.48^{* * *}$ & 1.12 \\
\hline Delayed memory & $100.19 \quad(13.40)$ & $105.31 \quad(14.00)$ & $0.55^{* *}$ & 5.12 & 0.38 \\
\hline Total Scale & $98.07 \quad(17.62)$ & 101.89 (16.07) & $0.80^{* * *}$ & 3.82 & 0.22 \\
\hline
\end{tabular}

RBANS: Repeatable Battery for the Assessment of Neuropsychological Status; CTT: Color Trails Test; mBNT: modified Boston Naming Test; SD: Standard Deviation. Effect Size: (Time 2 - Time 1) / Time 1's SD. * $p<0.05 ; * * p<0.01$; *** $p<0.001$ 


\subsection{Convergent Validity of the Tests}

As shown in Table 3, at time 1 the RBANS had a strong correlation with the composite $\mathrm{z}$ scores of RBANS supplemented with the CTT and mBNT $(r=.83, p<.001)$ while the strength of a significant correlation between the RBANS and a formal neuropsychological battery was relatively weaker ( $r=.61, p=.001)$. However, the correlation between the composite $\mathrm{z}$ scores of the RBANS supplemented with CTT and mBNT and the formal neuropsychological battery was strong $(r=.80, p$ $<.001)$.

\section{Discussion}

In this pilot study, we have examined the test-retest reliability, practice effects and convergent validity of a brief neuropsychological battery, the RBANS, in a sample of cognitively intact older adults in Singapore. The principal findings of this study include: 1) the RBANS has good test-retest reliability and convergent validity; and 2) the practice effects of the RBANS are evident in visuospatial function and memory domains.

The first hypothesis is supported, that is, the RBANS alone has demonstrated acceptable test-retest reliability for clinical purposes. In addition, RBANS supplemented with CTT and mBNT demonstrated excellent test-retest reliability. These results are consistent with the generally sound psychometric properties of the scales as previously reported. Test-retest reliability was also significant in 11 out of 12 RBANS subtests. Interestingly, the reliability coefficient for the remaining subtest (list recall) attained significance when three outlying cases were removed from the analysis. These three cases all scored a zero at time 1 , and scored highly at time 2, which may possibly reflect test anxiety affecting their performance at time 1 but not at time 2. Also of clinical interest is the finding of significant gain scores on many (but not all) of the RBANS subtests, while overall scores show little gain.

The second hypothesis was partially supported. The RBANS showed moderate to large practice effects in visuospatial function, immediate memory and delayed memory. The practice effect in immediate memory is consistent with a previous study on the practice effects of RBANS in a community sample of older adults in a

Table 3. Correlations of the total scale scores of RBANS, composite $Z$ scores of RBANS supplemented with CTT and mBNT and composite $Z$ scores of a formal neuropsychological battery.

\begin{tabular}{lcc}
\hline & 1 & 2 \\
\hline 1. RBANS & - & \\
2. RBANS with CTT and mBNT & $0.83^{* * *}$ & - \\
3. Formal Neuropsychological battery & $0.61^{* *}$ & $0.80^{* * *}$ \\
\hline$* * p<0.01 ; * * * p<0.001$. & &
\end{tabular}

${ }^{* *} p<0.01 ; * * * p<0.001$. western population[36]. However, the practice effects demonstrated in visuospatial function differed from the previous study[36], which may be explained by different sample characteristics such as age (younger participants in the current study compared to the older participants in previous study[36]: $63.29 \pm 5.84$ vs $72.89 \pm 5.52$ ). In addition, the different finding may be attributed to different test-retest interval (a short interval of 2 weeks in the cur-rent study compared to a longer interval of 38 weeks in the previous study [36]).

The third hypothesis was also confirmed. The RBANS showed adequate convergent validity with the formal neuropsychological battery. Moreover, the RBANS supplemented with CTT and mBNT has dem-onstrated excellent convergent validity. This result is encouraging, in that it suggests the RBANS may be an appropriate substitute for the lengthier neuropsychologi-cal test batteries in memory disorders clinics.

The novelty of this study includes the following. First, few studies to date have investigated the clinical utility of a brief neuropsychological test battery. The findings of this study support the use of the RBANS as an assessment instrument in clinical as well as research set-tings. The inclusion of CTT and mBNT further improves the psychometric properties such as test-retest reliability and convergent validity. Consequently, these two tests will add clinical value to the RBANS. This study has also reported valuable clinical data on the expected gain scores resulting from practice effects on the RBANS. This will provide insights for clinicians to observe for significant changes in raw subtest scores and index scores of RBANS. Second, a particular strength of this study is the complete data set, free from attrition which sometimes limits the ability to interpret results of repeated measures studies. However there were a number of ceiling effects identified in the data, which may have contributed to non-significant results. Such ceiling effects are to be expected in cognitively intact participants, yet reliability and validity of tests should be first established with cognitively intact participants prior to the implementation in clinical populations.

There are several limitations in this study. First, the sample size is small; we only recruited 30 cognitively intact participants with 28 participants included for analysis, as this was a pilot study. A larger sample size is required in future studies. Second, we did not examine the test-retest reliability, convergent validity and practice effects in older adults with cognitive impairment, again due to the nature of a pilot study. Older adults with cognitive impairment may have different rate of cognitive decline or improvement compared to healthy controls. Therefore, our findings are limited to cognitively intact older adults. Future studies should include older adults with a range of cognitive status to examine the test-retest 
reliability, convergent validity and practice effects of the RBANS. Third, the test-retest interval was short, at a span of 2 weeks, and may not be in line with clinical practice of repeat testing at 6 months or a year. Therefore, future studies with a longer span of test-retest duration will provide more information about the RBANS for clinical utility.

In conclusion, The RBANS has good test-retest reliability and convergent validity, while RBANS supplemented with CTT and mBNT have demonstrated excellent convergent validity. In addition, the practice effects were only demonstrated in immediate memory and visuospatial function while other index and total scale scores are spared of practice effects within the short span of a 2-week interval. Therefore, RBANS or RBANS supplemented with CTT and mBNT may be adopted for routine clinical practice in memory clinics.

\section{Acknowledgements}

This study is funded by a Memory Ageing Cognition Center (MACC) pilot grant (NMRC/CG/NUHS/ 2010) from the National Medical Research Council of Singapore. Y. Dong is currently receiving research support under the NMRC fellowship training award. The authors thank the research team from NUHS MACC for data collection and all participants for their involvement.

\section{REFERENCES}

[1] Alzheimer's Disease International, World Alzheimer Report 2009, London.

[2] S. H. Putnam and J. W. DeLuca, "The TCN Professional Practice Survey. Part I: General Practices of Neuropsychologists in Primary Employment and Private Practice Settings," The Clinical Neuropsychologist, Vol. 4, 1990, pp. 199-244. doi:10.1080/13854049008401 906

[3] M. McCrea, K. Guskiewicz, S. Marshall, W. Barr, C. Randolph, R. C. Cantu, et al., "Acute Effects and Recovery Time Following Concussion in Collegiate Football Players," Journal of the American Medical Association, Vol. 290, 2003, pp. 2556-2563.

doi:10.1001/ jama.290.19.2556

[4] C. Randolph, "Repeatable Battery for the Assessment of Neuropsychological Status (RBANS) Manual," The Psychological Corporation, San Antonio, TX, 1998.

[5] E. Strauss, E. M. Sherman and O. Spreen, "A Compendium of Neuropsychological Tests," 5th Edition, New York, Oxford University Press, 2006.

[6] K. Duff, J. D. Clark, S. E. O’Bryant, J. W. Mold, R. B. Schiffer and P. B. Sutker, "Utility of the RBANS in Detecting Cognitive Impairment associated with Alzheimer's Disease: Sensitivity, Specificity and Positive and Negative Predictive Powers," Archives Of Clinical Neuropsychology, Vol. 23, 2008, pp. 603-612. doi:10.1016/j.acn.2008.06.004
[7] K. Duff, V. L. Hobson, L. J. Beglinger and S. E. O’Bryant, "Diagnostic Accuracy of the RBANS in Mild Cognitive Impairment: Limitations on Assessing Milder Impairments," Archives of Clinical Neuropsychology, Vol. 25, 2010, pp. 429-441. doi:10.1093/arclin/acq045

[8] C. Garcia, B. Leahy, K. Corradi and C. Forchetti, "Component Structure of the Repeatable Battery for the Assessment of Neuropsychological Status in Dementia," Archives Of Clinical Neuropsychology, Vol. 23, 2008, pp. 63-72. doi:10.1016/j.acn.2007.08.008

[9] L. F. D'Elia, P. Satz, C. Uchiyama and T. White, "Color Trails Test Professional Manual," Psychological Assessment Resources, Odessa, FL, 1996. doi:10.1097/00002093-200204000-00003

[10] S. J. Sahadevan, P. P. Lim, et al., "Psychometric Identification Of Early Alzheimer's Disease In An Elderly Chinese Population With Differing Educational Levels," Alzheimer's Disease and Associated Disorders, Vol. 16, 2002, pp. 65-72. doi:10.1097/00002093- 200204000-00003

[11] M. Lezak, D. Howieson and D. Loring, "Neuropsychological Assessment," 4th Edition, Oxford University Press, New York, 2004.

[12] M. G. Falleti, P. Maruff, A. Collie and D. G. Darby, "Practice Effects Associated with the Repeated Assessment of Cognitive Function using the Cogstate Battery at 10-Minute, One Week and One Month Test-Retest Intervals," Journal Of Clinical And Experimental Neuropsychology, Vol. 28, 2006, pp. 1095-1112.

doi:10.1080/13803390500205718

[13] J. Lu, D. Li, F. Li, A. Zhou, F. Wang, X. Zuo, X. Jia, H. Song and J. Jia. "Montreal Cognitive Assessment in Detecting Cognitive Impairment in Chinese Elderly Individuals: A Population-Based Study," Journal of Geriatric Psychiatry And Neurology, Vol. 24, 2011, pp. 184-190. doi:10.1177/0891988711422528

[14] C. Memoria, P. Bertolucci, A. L. Sarmento, J. R. Wajman and O. Forlenza, "Brief Screening for Mild Cognitive Impairment: Initial Validation of the Montreal Cognitive Assessment (MOCA) in Brazilian Elderly," Alzheimer's And Dementia, Vol. 7, 2011, p. s256.

doi:10.1016/j.jalz.2011.05.726

[15] D. E. Patton, K. Duff, M. R. Schoenberg, J. Mold, J. G. Scott and R. L. Adams, "Base Rates of Longitudinal RBANS Discrepancies At One- And Two-Year Intervals In Community-Dwelling Older Adults," The Clinical Neuropsychologist, Vol. 19, No. 1, 2005, pp. 27-44. doi:10.1080/13854040490888477.

[16] D. Yeo, C. Gabriel, C. Chen, S. Lee, T. Loenneker and M. Wong, "Pilot Validation of a Customized Neuropsychological Battery in Elderly Singaporeans," Neurological Journal of South East Asia, Vol. 2, 1997, p. 123.

[17] K. Duff, W. R. Leber, D. E. Patton, M. R. Schoenberg, J. W. Mold, J. G. Scott and R. L. Adams, "Modified Scoring Criteria for the RBANS Figures," Applied Neuropsychology, Vol. 14, 2007, pp. 73-83.

doi:10.1080/09084280701319805 
[18] M. L. Lim, S. L. Collinson, L. Feng and T. P. Ng, Cross-Cultural Application of the Repeatable Battery for the Assessment of Neuropsychological Status (RBANS): Performances of Elderly Chinese Singaporeans. Clinical Neuropsychologist, Vol. 24, 2010, pp. 811-826. doi:10.1080/13854046.2010.490789

[19] L. F. D'Elia, P. Satz, C. Uchiyama and T. White,). "Color Trails Test, Professional Manual," Psychological Assessment Resources, Odessa, FL, 1996.

[20] D. Wechsler, "Wechsler Memory Scale-Revised," Harcourt Brace, San Antonio, TX, 1997.

[21] R. F. Lewis and P. M. Rennick, "Manual for the Repeatable Cognitive Perceptual-Motor Battery," Axon, Clinton Township, MI, 1979.

[22] W. J. Mack, D. M. Freed, B. W. Williams et al., "Boston Naming Test: Shortened Versions for use in Alzheimer's disease," Journal of Gerontology, Vol. 47, 1992, pp. 154-158. doi:10.1093/geronj/47.3.P154

[23] B. Isaacs and A. T. Kennie, "The Set Test as an Aid to the Detection of Dementia in Old People," British Journal of Psychiatry, Vol. 123, 1973, pp. 467-470. doi:10.1192/bjp.123.4.467

[24] A. Smith, "Symbol Digit Modalities Test," Western Psychological Services, Los Angeles, CA, 1973.

[25] L. Diller, Y. Ben-Yishay, L. J. Gerstman et al., "Studies in Cognition and Rehabilitation in Hemiplegia," Rehabilitation Monograph, No. 50, 1974, University Medical Center, New York.

[26] S. D. Porteus. "The Maze Test and Clinical Psychology," Pacific Books, Palo Alto, CA, 1959.

[27] T. Sunderland, J. L. Hill, A. M. Mellow et al., "Clock Drawing in Alzheimer's Disease. A Novel Measure of Dementia Severity," Journal of the American Geriatric Society, Vol. 37, 1989, pp. 725-729.

[28] D. Wechsler. "Wechsler Adult Intelligence Scale -Revised," Harcourt Brace, New York, 1981.

[29] B, Dubois, I. Litvan, "The FAB: A Frontal Assessment
Battery at Bedside," Neurology, Vol 55, 2000, pp. 1621-1626. doi:10.1212/WNL.55.11.1621

[30] H. M. Hodkinson, "Evaluation of a Mental Test Score for Assessment of Mental Impairment in the Elderly," Age and Aging, Vol. 1, 1972, pp. 233-238.

doi:10.1093/ageing/1.4.233

[31] M. F. Folstein, S. E. Folstein and P. R. McHugh, "Mini-Mental State. A Practical Method for Grading the Cognitive State of Patients for the Clinician," Journal of Psychiatric Research, Vol. 12, 1975, pp. 189-198. doi:10.1016/0022-3956(75)90026-6

[32] Z. S. Nasreddine, N. A. Phillips, V. Bedirian, S. Charbonneau, V. Whitehead, I. Collin et al., "The Montreal Cognitive Assessment, MOCA: A Brief Screening Tool for Mild Cognitive Impairment," Journal of the American Geriatrics Society, Vol. 53, 2005, pp. 695-699. doi:10.1111/j.1532-5415.2005.53221.x

[33] J. I. Sheikh and J. A Yesavage, "Geriatric Depression Scale (GDS): Recent Evidence and Development of a Shorter Version," In: T. L. Brink Ed., Clinical Gerontology: A Guide to Assessment and Intervention, The Haworth Press, New York, 1986, pp. 165-173.

[34] J. Cohen, "A Power Primer," Psychological Bulletin, Vol. 112, 1992, pp. 155-159. doi:10.1037/ 0033-2909.112.1.155

[35] S. S. Dikmen, R. K Heaton, I. Grant and N. R. Temkin, "Test-Retest Reliability and Practice Effects of Expanded Halstead-Reitan Neuropsychological Test Battery," Journal of the International Neuropsychological Society, Vol. 5, 1999, pp. 346-359. doi:10.1017/ S1355617799544056

[36] K. Duff, L. J. Beglinger, M. R. Schoenberg, D. E. Patton, J. Mold, J. G. Scott et al., "Test-Retest Stability and Practice Effects of the RBANS in a Community Dwelling Elderly Sample," Journal of Clinical and Experimental Neuropsychology, Vol. 27, 2005, pp. 565-575.

doi:10.1080/13803390490918363 